\title{
GAUGE THEORIES FOR GRAVITY ON A LINE*
}

\author{
R. Jackiw \\ Center for Theoretical Physics \\ Laboratory for Nuclear Science \\ and Department of Physics \\ Massachusetts Institute of Technology \\ Cambridge, Massachusetts 02139 U.S.A.
}

Submitted to: Theoretical and Mathematical Physics

In Memoriam

M. C. Polivanov (D.O.E.) under contract \#DE-AC02-76ER03069. 


\section{INTRODUCTION}

Professor M. C. Polivanov and I met only a few times, during my infrequent visits to thethen Soviet Union in the 1970's and 1980's. His hospitality at the Moscow Steclov Institute made the trips a pleasure, while the scientific environment that he provided made them professionally valuable. But it is the human contact that I remember most vividly and shall now miss after his death. At a time when issues of conscience were both pressing for attention and difficult/dangerous to confront, Professor Polivanov made a deep impression with his quiet but adamant commitment to justice. I can only guess at the satisfaction he must have felt when his goal of gaining freedom for Yuri Orlov was attained, and even more so these days when human rights became defensible in his country; it is regrettable that he cannot now enjoy the future that he strived to attain.

One of our joint interests was the Liouville theory, ${ }^{1,2}$ which in turn can be viewed as a model for gravity in two-dimensional space-time. Some recent developments in this field are here summarized and dedicated to Polivanov's memory, with the hope that he would have enjoyed knowing about them.

We study lower-dimensional gravity both for pedagogical reasons - one expects that the dimensional reduction effects sufficient simplification to permit thorough analysis, while still retaining useful content to inform the physical $(3+1)$-dimensional problem - and also, if one is lucky, there are practical applications - e.g. idealized cosmic strings are described by $(2+1)$-dimensional gravity, while the still lower-dimensional models are used in statistical mechanics.

The drastic dimensional reduction to $(1+1)$ dimensions - gravity on a line, i.e., lineal gravity - is not devoid of interest, provided dynamical equations are not based on the Einstein

tensor $G_{\mu \nu}=R_{\mu \nu}-\frac{1}{2} g_{\mu \nu} R$, which vanishes identically in two dimensions. 
In a proposal of several years ago, ${ }^{3}$ it was suggested that gravity equations be based on the Riemann scalar $R$, the simplest entity that encodes in two dimensions all local geometric information about space-time. Moreover, in an action formulation it is necessary to introduce an additional scalar field, which acts as a Lagrange multiplier that enforces the equation of motion for $R$. Thus we are dealing with scalar-tensor theories, or - to use the contemporary string nomenclature — "dilaton" gravities.

Since the initial proposal, various models have been studied. Here I shall describe two that are selected by their group theoretical properties: they can be formulated as gauge theories based on groups relevant to space-time: de Sitter or anti-de Sitter (in $(1+1)$-dimensions both groups are $S O(2,1)$, although the geometries are different) and Poincaré. The first of these is the one proposed originally; ${ }^{3}$ it is governed by the action

$$
I_{1}=\int d^{2} x \sqrt{-g} \eta(R-\Lambda)
$$

The second is "string-inspired" and has been recently studied for purposes of modeling (on a line!) black hole physics; ${ }^{4}$ its action is

$$
\bar{I}_{2}=\int d^{2} x \sqrt{-\bar{g}} e^{-2 \varphi}\left(\bar{R}-4 \bar{g}^{\mu \nu} \partial_{\mu} \varphi \partial_{\nu} \varphi-\Lambda\right)
$$

(Notation: time and space carry the metric tensor $g_{\mu \nu}$ with signature $(1,-1)$. The twovector $x^{\mu}=(t, x)$ will be frequently presented in light-cone components $x^{ \pm} \equiv \frac{1}{\sqrt{2}}(t \pm x)$. Tangent space components are labeled by Latin letters $a, b, \ldots$, and the Minkowski metric tensor $h_{a b}=\operatorname{diag}(1,-1)$ raises/lowers these indices. Also we use the anti-symmetric tensor $\left.\epsilon^{a b}, \epsilon^{01}=1.\right)$

In (1), $R$ is the scalar curvature built from $g_{\mu \nu}, \eta$ is a world scalar Lagrange multiplier related to the dilaton, while $\Lambda$ is a cosmological constant. In $(\overline{2})$ we temporarily use an overbar to denote a differently scaled metric tensor $\bar{g}_{\mu \nu}$ from which $\bar{R}$ is constructed, while $\varphi$ is 
the dilaton. Formula $(\overline{2})$ arises naturally from string theory, restricted to a two-dimensional target space, with the anti-symmetric tensor field identically vanishing. In the string context, matter is taken to couple to $\bar{g}_{\mu \nu}$; for our purposes in the absence of matter it is convenient to redefine variables by $\bar{g}_{\mu \nu}=e^{2 \varphi} g_{\mu \nu}, \eta=e^{-2 \varphi}$. Then $(\overline{2})$ becomes

$$
I_{2}=\int d^{2} x \sqrt{-g}(\eta R-\Lambda)
$$

but it is to be remembered that because of the redefinition, the "physical" metric tensor is $g_{\mu \nu} /(-2 \eta)$. Note that $(2)$ is invariant against shifting $\eta$ by a constant, because $\sqrt{-g} R$ is a total derivative.

It is seen that the two models (1) and (2) differ in the placement of the Lagrange multiplier with the cosmological term: in (1) $\eta$ multiplies $\Lambda$, in (2) the $\eta$ factor is absent from $\Lambda$. Of course in the limit $\Lambda=0$, the difference disappears.

We now describe the interesting gauge group structure of (1) and (2) which we name (anti) de Sitter gravity and extended Poincaré gravity, respectively.

\section{II. (ANTI) DE SITTER GRAVITY}

The equations of motion that follow from varying $\eta$ and $g_{\mu \nu}$ in (1) are

$$
\begin{gathered}
R=\Lambda \\
\left(\mathcal{D}_{\mu} \mathcal{D}_{\nu}-g_{\mu \nu} \mathcal{D}^{2}\right) \eta+\frac{\Lambda}{2} g_{\mu \nu} \eta=0
\end{gathered}
$$

The second equation, with $\mathcal{D}_{\mu}$ the space-time covariant derivative, can be decomposed into traceless and trace parts.

$$
\begin{aligned}
\left(\mathcal{D}_{\mu} \mathcal{D}_{\nu}-\frac{1}{2} g_{\mu \nu} \mathcal{D}^{2}\right) \eta & =0 \\
\left(\mathcal{D}^{2}-\Lambda\right) \eta & =0
\end{aligned}
$$


The above geometric dynamics may be presented in a gauge theoretical fashion. ${ }^{5}$ To this end one uses the (anti) de Sitter group with Lorentz generator $J$ and translation generators $P_{a}$ satisfying the $S O(2,1)$ algebra $($ for $\Lambda \neq 0)$.

$$
\left[P_{a}, J\right]=e_{a}{ }^{b} P_{b}, \quad\left[P_{a}, P_{b}\right]=-\frac{\Lambda}{2} \epsilon_{a b} J
$$

The gauge connection one-form is introduced $A=A_{\mu} d x^{\mu}$ and expanded in terms of the generators,

$$
A=e^{a} P_{a}+\omega J
$$

where $e_{\mu}^{a}$ is the Zweibein and $\omega_{\mu}$ is the spin-connection. The curvature two-form

$$
F=d A+A^{2}
$$

becomes

$$
\begin{gathered}
F=f^{a} P_{a}+f J=(D e)^{a} P_{a}+\left(d \omega-\frac{\Lambda}{4} e^{a} \epsilon_{a b} e^{b}\right) J \\
(D e)^{a} \equiv d e^{a}+\epsilon^{a}{ }_{b} \omega e^{b}
\end{gathered}
$$

It is seen that $d \omega$ is the scalar curvature density and $f^{a}$ is the torsion density, each expressed in terms of $e^{a}$ and $\omega$, which at this stage are independent variables.

The Lagrange density

$$
\begin{aligned}
\mathcal{L}_{1}^{\prime} & =\sum_{A=0}^{2} \eta_{A} F^{A}=\eta_{a}(D e)^{a}+\eta_{2}\left(d \omega-\frac{\Lambda}{4} e^{a} \epsilon_{a b} e^{b}\right) \\
F^{A} & =\left(f^{a}, f\right), \quad \eta_{A}=\left(\eta_{a}, \eta_{2}\right)
\end{aligned}
$$

is gauge invariant: the three field strengths $F^{A}$ transform covariantly according to the threedimensional adjoint representation, while the Lagrangian multiplet triplet $\eta_{A}$ transforms by the coadjoint representation. 
The equation obtained from (10) by varying $\eta_{a}$ gives the condition of vanishing torsion, and allows evaluating the spin connection in terms of the Zweibein.

$$
\omega=e^{a}\left(h_{a b} \epsilon^{\mu \nu} \partial_{\mu} e_{\nu}^{b}\right) / \operatorname{det} e
$$

The equation which follows upon variation of $\eta_{2}$ regains (3) once (11) is used. Variation of $e^{a}$ and $\omega$ produces equations for the Lagrange multipliers $\eta_{a}$ and $\eta_{2}$, respectively, the latter of course coinciding with $\eta$ in the geometric formulations (1), (3) and (4).

$$
\begin{aligned}
d \eta_{a}+\epsilon_{a}{ }^{b} \omega \eta_{b}-\frac{\Lambda}{2} \epsilon_{a b} \eta_{2} e^{b} & =0 \\
d \eta_{2}+\eta_{a} \epsilon^{a}{ }_{b} e^{b} & =0
\end{aligned}
$$

Upon taking a space-time covariant derivative of (12b) and using (12a) to eliminate $\eta_{a}$, we recover (4). Finally we see that when $\omega$ is eliminated from $\mathcal{L}_{1}^{\prime}$ with the help of (11), so that the torsion (9) vanishes, what remains is the Lagrange density of (1), expressed in terms of Zweibeine.

Thus the geometric formulation of this gravity theory is contained within the (anti) de Sitter group theoretical framework for solutions with $\operatorname{det} e \neq 0$, but see below.

Explicit classical solutions to the equations are easy to find. Working within the geometric framework, we use coordinate invariance to choose a conformally flat metric tensor.

$$
g_{\mu \nu}=h_{\mu \nu} \exp 2 \sigma
$$

Then (3) becomes the Liouville equation,

$$
\square \sigma=\frac{\Lambda}{2} \exp 2 \sigma
$$


studied by Polivanov. ${ }^{1}$ Its general solution depends on two arbitrary functions of the two light-cone variables, $F\left(x^{+}\right), G\left(x^{-}\right)$,

$$
\exp 2 \sigma=\frac{F^{\prime}\left(x^{+}\right) G^{\prime}\left(x^{-}\right)}{\left(1-\frac{\Lambda}{4} F G\right)^{2}}
$$

whose derivatives fulfill the consistency condition $F^{\prime} G^{\prime}>0$. But the residual coordinate invariance within the conformal gauge allows choosing $F\left(x^{+}\right)=x^{+}, G\left(x^{-}\right)=x^{-}$, hence

$$
\exp 2 \sigma=\frac{1}{\left(1-\frac{\Lambda}{8} x^{2}\right)^{2}}
$$

In conformal gauge, $(4 \mathrm{~b})$ reduces to

$$
\partial_{\mu} V_{\nu}+\partial_{\nu} V_{\mu}-h_{\mu \nu} h^{\alpha \beta} \partial_{\alpha} V_{\beta}=0
$$

where $V_{\mu}$ is defined by

$$
V_{\mu} \exp 2 \sigma=\partial_{\mu} \eta
$$

Equation (17) is just the (flat-space) conformal Killing equation with solutions in terms of arbitrary functions of a single light-cone variable.

$$
V_{-}=V_{-}\left(x^{+}\right), \quad V_{+}=V_{+}\left(x^{-}\right)
$$

Finally the remaining equation (4c) together with (18) restricts these functions, so that the solution for $\eta$ takes the form

$$
\eta=\frac{\alpha_{a} x^{a}+\alpha_{2}\left(1+\frac{\Lambda}{8} x^{2}\right)}{1-\frac{\Lambda}{8} x^{2}}
$$

where $\alpha_{a}$ is a constant two-vector and $\alpha_{2}$ is a constant scalar. 
The Zweibein and spin connection of the gauge theoretical formulation are given by related formulas. The former, the "square root" of the metric tensor, becomes (apart from an arbitrary Lorentz transformation on the tangent-space indices)

$$
e_{\mu}^{a}=\delta_{\mu}^{a} \exp \sigma=\frac{1}{1-\frac{\Lambda}{8} x^{2}} \delta_{\mu}^{a}
$$

while the latter is

$$
\omega_{\mu}=-h_{\mu \alpha} \epsilon^{\alpha \beta} \partial_{\beta} \sigma
$$

The Lagrange multiplier $\eta_{2}$ coincides with $\eta$, while Eq. (12) for $\eta_{a}$ is solved by

$$
\eta_{a} \exp \sigma=\epsilon_{a}{ }^{\mu} \partial_{\mu} \eta
$$

Of course the general solution is an arbitrary coordinate transformation of the above.

Finally we observe that the gauge theoretical formulation allows an alternative group theoretical presentation of solutions. The field equations following from (10), upon respective variation of $\eta_{A}$ and $A$, are

$$
\begin{gathered}
F=0 \\
d H+[A, H]=0
\end{gathered}
$$

$A, F$ and $H=\eta_{a} h^{a b} P_{b}+\frac{2}{\Lambda} \eta_{2} J$ belong to the $S O(2,1)$ algebra (the factor $2 / \Lambda$ is a consequence of the group metric). Equation (24) implies that $A$ is a pure gauge given by an arbitrary element $U$ of the $S O(2,1)$ group,

$$
A=U^{-1} d U
$$

while the Lagrange multiplier is then determined by (25) to be

$$
H=U^{-1} \Phi U
$$


where $\Phi$ is a constant element in the algebra. The explicit group and algebra elements that correspond to the above solution, Eqs. (20) - (23), are

and

$$
U=\exp \left(\frac{i \pi}{\sqrt{1-\frac{\Lambda}{8} x^{2}}}\left(-\frac{1}{2} x^{a} \epsilon_{a}{ }^{b} P_{b}+J\right)\right)
$$

$$
\Phi=\frac{2}{\Lambda} \alpha_{a} \epsilon^{a b} P_{b}-\alpha_{2} J
$$

$U$ is unique up to a constant gauge transformation.

Within the gauge theoretical framework, an even simpler solution to (24) and (25) is available: $A=0, H=\Phi$, which makes no sense geometrically: not only $\operatorname{det} e$, but both the connections $e^{a}$ and $\omega$ vanish! But in fact use can be made of such solutions: when presented with a geometrically singular configuration, perform any gauge transformation producing non-singular connections, for example with the group element $U$ above. So we see that the group theoretical framework, even in its $\operatorname{det} e=0$ sector, contains adequate information for encoding the gravity theory.

\section{EXTENDED POINCARÉ GRAVITY}

Equations of motion of the string-inspired gravitational theory (2) are, from varying $\eta$

$$
R=0
$$

and from varying $g_{\mu \nu}$

$$
\left(\mathcal{D}_{\mu} \mathcal{D}_{\nu}-g_{\mu \nu} \mathcal{D}^{2}\right) \eta+\frac{\Lambda}{2} g_{\mu \nu}=0
$$

which is equivalent to

$$
\mathcal{D}_{\mu} \mathcal{D}_{\nu} \eta=\frac{\Lambda}{2} g_{\mu \nu}
$$


Note that (31a) differs from (4a) by the absence of $\eta$ in the last term.

To give a gauge theoretical formulation, ${ }^{6}$ we make use of the centrally extended Poincaré group, whose algebra is

$$
\left[P_{a}, J\right]=\epsilon_{a}^{b} P_{b} \quad, \quad\left[P_{a}, P_{b}\right]=\epsilon_{a b} I
$$

where the central element $I$ commutes with $P_{a}$ and $J$. Consequently the connection $A$ and curvature $F$ now become

$$
\begin{aligned}
A & =e^{a} P_{a}+\omega J+a I \\
F & =d A+A^{2}=f^{a} P_{a}+f J+g I \\
& =(D e)^{a} P_{a}+d \omega J+\left(d a+\frac{1}{2} e^{a} \epsilon_{a b} e^{b}\right) I
\end{aligned}
$$

Here $a$ and $g$ are the additional connection and curvature associated with the central element in the algebra.

This magnetic-like extension of the Poincaré group may be viewed as an unconventional contraction of the de Sitter group: The ordinary Poincaré algebra (Eq. (32) without the central element) is the $\Lambda \rightarrow 0$ contraction of the $S O(2,1)$ algebra (5). However, owing to the well-known ambiguity of two-dimensional angular momentum, in (5) one may replace $J$ by $J-2 I / \Lambda$ before taking the $\Lambda \rightarrow 0$ limit, which then leaves (32).

The extension reflects a 2-cocycle in the composition law for representatives of the Poincaré group. If the group acts on coordinates $x^{a}$ by

$$
x^{a} \longrightarrow \bar{x}^{a}=\mathcal{M}^{a}{ }_{b} x^{b}+q^{a}
$$

where $\mathcal{M}$ is a finite Lorentz transformation

$$
\mathcal{M}^{a}{ }_{b}=\delta^{a}{ }_{b} \cosh \alpha+\epsilon^{a}{ }_{b} \sinh \alpha
$$


and $q^{a}$ is a finite translation, the composition law for these is

$$
\begin{aligned}
\mathcal{M}_{(12)} & =\mathcal{M}_{1} \mathcal{M}_{2} \\
q_{(12)} & =q_{1}+\mathcal{M}_{1} q_{2}
\end{aligned}
$$

However, the composition law for a representation $G(\mathcal{M}, q)$ containing the extension (32) in its algebra acquires a 2-cocycle.

$$
G\left(\mathcal{M}_{1}, q_{1}\right) G\left(\mathcal{M}_{2}, q_{2}\right)=\exp \left\{\frac{i}{2} q_{1}^{a} \epsilon_{a b}\left(\mathcal{M}_{1} q_{2}\right)^{b}\right\} G\left(\mathcal{M}_{1} \mathcal{M}_{2}, q_{1}+\mathcal{M}_{1} q_{2}\right)
$$

( $I$ is represented by $i=\sqrt{-1}$.)

A finite gauge transformation, generated by the gauge function $\Theta$,

$$
\Theta=\theta^{a} P_{a}+\alpha J+\beta I
$$

produces the following transformations on the connections.

$$
\begin{aligned}
e^{a} \rightarrow \bar{e}^{a} & =\left(\mathcal{M}^{-1}\right)^{a}{ }_{b}\left(e^{b}+\epsilon_{c}^{b} \theta^{c} \omega+d \theta^{b}\right) \\
\omega \rightarrow \bar{\omega} & =\omega+d \alpha \\
a \rightarrow \bar{a} & =a-\theta^{a} \epsilon_{a b} e^{b}-\frac{1}{2} \theta^{2} \omega+d \beta+\frac{1}{2} d \theta^{A} \epsilon_{a b} \theta^{b}
\end{aligned}
$$

The multiplet of curvatures $F^{A}=\left(f^{a}, f, g\right)$ transforms by the adjoint $4 \times 4$ representation of the extended group,

$$
\begin{aligned}
f^{a} \rightarrow \bar{f}^{a} & =\left(\mathcal{M}^{-1}\right)_{b}^{a}\left(f^{b}+\epsilon^{b}{ }_{c} \theta^{c} f\right) \\
f \rightarrow \bar{f} & =f \\
g & \rightarrow \bar{g}=g-\theta^{a} \epsilon_{a b} f^{b}-\frac{1}{2} \theta^{2} f
\end{aligned}
$$

or

$$
\begin{aligned}
F^{A} \rightarrow \bar{F}^{A} & =\sum_{B=0}^{3}\left(U^{-1}\right)_{B}^{A} F^{B} \\
U & =\left(\begin{array}{ccc}
\mathcal{M}^{a}{ }_{b} & -\epsilon^{a}{ }_{c} \theta^{c} & 0 \\
0 & 1 & 0 \\
\theta^{c} \epsilon_{c d} \mathcal{M}_{b}^{d} & -\theta^{2} / 2 & 1
\end{array}\right)
\end{aligned}
$$


The upper left $3 \times 3$ block in $U$ comprises the adjoint representation of the conventional Poincaré group with $q^{a}$ of (35) identified with $-\epsilon_{c}^{a} \theta^{c}$, while the fourth row and column arise from the extension. Note that in the above realization of the gauge action on $F$, the extension is not visible: $I$ is represented by $\mathbf{O}$. On the other hand, an additional connection and curvature $(a, g)$ are present.

In this representation, the extended algebra possesses a non-singular Killing metric, which is unavailable without the extension.

$$
h_{A B}=\left(\begin{array}{crr}
h_{a b} & 0 & 0 \\
0 & 0 & -1 \\
0 & -1 & 0
\end{array}\right)
$$

It is true that ${ }^{T} U h U=h$; this allows raising and lowering the indices $(A, B)$.

An invariant Lagrange density is now constructed with an extended multiplet of Lagrange multipliers $\eta_{A}$,

$$
\begin{aligned}
\mathcal{L}_{2}^{\prime} & =\sum_{A=0}^{3} \eta_{A} F^{A}=\eta_{a}(D e)^{a}+\eta_{2} d \omega+\eta_{3}\left(d a+\frac{1}{2} e^{a} \epsilon_{a b} e^{c}\right) \\
F^{A} & =\left(f^{a}, f, g\right), \quad \eta_{a}=\left(\eta_{a}, \eta_{2}, \eta_{3}\right)
\end{aligned}
$$

which obey the coadjoint transformation law,

$$
\eta_{A} \rightarrow \bar{\eta}_{A}=\sum_{B=0}^{3} \eta_{B} U_{A}^{B}
$$

or in components

$$
\begin{aligned}
& \eta_{a} \rightarrow \bar{\eta}_{a}=\left(\eta_{b}-\eta_{3} \epsilon_{b c} \theta^{c}\right) \mathcal{M}_{a}^{b} \\
& \eta_{2} \rightarrow \bar{\eta}_{2}=\eta_{2}-\eta_{a} \epsilon_{b}^{a} \theta^{b}-\frac{1}{2} \eta_{3} \theta^{2} \\
& \eta_{3} \rightarrow \bar{\eta}_{3}=\eta_{3}
\end{aligned}
$$

Using the invariant metric (42), other group invariants may be constructed.

$$
\begin{aligned}
\mathcal{F}^{2} & =\sum_{A, B=0}^{3} * F^{A} h_{A B} F^{B} \\
M & =-\frac{2}{\Lambda} \sum_{A, B=0}^{3} \eta_{A} h^{A B} \eta_{B}
\end{aligned}
$$


where ${ }^{*} F^{A}$ is the 0 -form $\frac{1}{2} \epsilon^{\mu \nu} F_{\mu \nu}^{A}$, dual to the 2 -form $F^{A}$.

We recognize in (43) the torsion $(D e)^{a}$ and curvature $d \omega$ densities, which vanish as a consequence of varying $\eta_{a}$ and $\eta_{2}$, respectively. Thus Eq. (30) is regained. The Lagrange multiplier $\eta$ in (2) corresponds to $\eta_{2}$ in the present formulas and the equation for it, obtained by varying $\omega$, is as in the (anti) de Sitter model, (12b),

$$
d \eta_{2}+\eta_{a} \epsilon_{b}^{a} e^{b}=0
$$

while the equation for $\eta_{a}$, obtained by varying $e^{a}$, differs from (12a),

$$
d \eta_{a}+\epsilon_{a}^{b} \omega \eta_{b}+\eta_{3} \epsilon_{a b} e^{b}=0
$$

We need a value for $\eta_{3}$ to close the system (48). The equation for that multiplier is obtained by varying $a$,

$$
d \eta_{3}=0
$$

and a constant, cosmological solution

$$
\eta_{3}=-\frac{\Lambda}{2}
$$

renders (48b) similar to (12a),

$$
d \eta_{a}+\epsilon_{a}{ }^{b} \omega \eta_{b}-\frac{\Lambda}{2} \epsilon_{a b} e^{b}=0
$$

except that there is no factor of $\eta_{2}$ in the last, cosmological term of (48e). This of course has the consequence that when (48a) and (48e) are combined as before, the second order equation that emerges for $\eta=\eta_{2}$ reproduces (31). 
The remaining equation of the gauge theoretical formulation, obtained by varying $\eta_{3}$

$$
d a=-\frac{1}{2} e^{a} \epsilon_{a b} e^{b}
$$

and allowing evaluation of $a$, has no counterpart in the geometric formulation. Equation (49) can always be locally integrated because the right side is a two-form, hence closed in two dimensions. However in general, there will be singularities in $a$, since upon integrating (49) over a two-space, the right side gives the total "volume," which could be a well-defined non-vanishing quantity, while the left side always integrates to zero if the manifold is closed and bounded, and $a$ is non-singular.

Note that upon eliminating $\omega$ in $\mathcal{L}_{2}^{\prime}$ with the zero-torsion equation $(D e)^{a}=0$ and evaluating $\eta_{3}$ at $-\Lambda / 2, \mathcal{L}_{2}^{\prime}$ coincides with the Lagrange density in (2), now expressed in terms of Zweibeine, apart from the total derivative $-\Lambda / 2 d a$, which does not contribute to equations of motion.

Thus here again, the group theoretical formulation reproduces the geometric one, for solutions with det $e \neq 0$, but again see below. However, the former is more flexible: Eq. (48c) is satisfied with vanishing $\eta_{3}$; this corresponds to a vanishing cosmological constant. Thus the gauge theory built on the extended Poincaré group possesses as a solution a non-extended system. It is interesting therefore that here the cosmological term is an integration constant, and not inserted a priori into the theory.

Finding explicit solutions is straightforward. In the geometric formulation, (3) is solved by a flat metric tensor.

$$
g_{\mu \nu}=h_{\mu \nu}
$$

Then (31) immediately gives

$$
-2 \eta=M-\frac{\Lambda}{2}\left(x-x_{0}\right)^{2}
$$


with $M$ and $x_{0}$ being integration constants, the former reflecting the $\eta$-translation invariance mentioned earlier.

Interest in the model ${ }^{4}$ derives precisely from the above "black-hole" solution with mass $M$ [in terms of the "physical" metric $\left.g_{\mu \nu} /(-2 \eta)\right]$, located at $x_{0}$. An arbitrary coordinate transformation of this configuration produces the general solution.

The gauge theoretical counterparts of the above are a flat Zweibein (apart from a constant tangent-space Lorentz transformation)

$$
e_{\mu}^{a}=\delta_{\mu}^{a}
$$

and a vanishing spin connection.

$$
\omega=0
$$

Taking in (48c) the cosmological solution for $\eta_{3}$, allows solving (48e) for $\eta_{a}$

$$
\eta_{a}=\frac{\Lambda}{2} \epsilon_{a \mu}\left(x^{\mu}-x_{0}^{\mu}\right)
$$

and from (48a) $\eta_{2}=\eta$ is recovered to be as in (51). Finally (49) is solved for $a$.

$$
a_{\mu}=\frac{1}{2} \epsilon_{\mu \nu} x^{\nu}
$$

with a pure gauge contribution $\partial_{\mu} \chi$ left arbitrary. The potential in (55) corresponds to a constant "magnetic field," as is appropriate with our "magnetic-like" extension of translations.

Note the two invariants defined in (46) and (47): $\mathcal{F}^{2}$ vanishes since $F^{A}$ does, while $M$ is recognized as the "black hole" mass.

The gauge theoretical solution may of course also be presented in a group theoretical fashion, since the equations are of the same form as in (24) and (25), with all quantities 
belonging to the extended algebra and group. The explicit formulas, corresponding to the "black hole" solution, Eqs. (50) - (55), are as follows. The group element $U$ that leads to the pure gauge connection $A=U^{-1} d U$ is

$$
U=\exp x^{a} P_{a}
$$

up to a constant gauge transformation. The constant algebra element $\Phi$ that gives $H=$ $\eta_{a} h^{a b} P_{b}-\eta_{3} J-\eta_{2} I=U^{-1} \Phi U$ is (placement of $\eta_{2}$ and $\eta_{3}$ dictated by the group metric (42), viz. $\left.\eta^{A}=h^{A B} \eta_{B}\right)$

$$
\Phi=\frac{\Lambda}{2} x_{0}^{a} \epsilon_{a}{ }^{b} P_{b}+\frac{\Lambda}{2} J+\left(\frac{M}{2}-\frac{\Lambda}{4} x_{0}^{2}\right) I
$$

As in the (anti) de Sitter model, we see that after a further gauge transformation we pass to the geometrically singular configuration $A=0, H=\Phi$. This gives an especially succinct account of the relevant geometric information : $\Phi$ encodes the integration constants, which characterize the intrinsic geometry: the cosmological constant $\Lambda$, the "black hole" mass $M$ and location $x_{0}$. A geometry is built with these characteristics once a gauge transformation is performed, say with the above $U$, to obtain non-singular connections.

\section{CONCLUSION}

The two models here considered are special: their geometric dynamics possess a gauge theoretical formulation. The extended Poincaré model exhibits the intriguing possibility of a cosmological term that is an integration constant, as are the "black hole" mass $M$ and location $x_{0}$; all three are encoded in the Lagrange multipliers of the theory.

Both models can also be obtained by dimensional reduction from $(2+1)$ dimensions: To obtain (anti) de Sitter gravity in its geometric formulation one begins ${ }^{3}$ with the Einstein 
theory/Hilbert action (with cosmological term), suppresses dependence on the third dimension, sets $g_{\mu 2}$ to zero for $\mu=0,1$ and $g_{22}$ to $\eta^{2}$; for the gauge theoretical formulation one starts with the Dreibein-spin connection form of the theory, which also is equivalent to a Chern-Simons, $\mathcal{O}(2,2)$ or $\mathcal{O}(3,1)$ model. ${ }^{7}$ Extended Poincaré gravity can be similarly constructed, but the higher-dimensional theory has to be suitably extended by an Abelian ideal. Indeed it is found that both the (anti) de Sitter and extended Poincaré $(1+1)$ dimensional theories arise as different dimensional reductions of a single, extended $(2+1)$-dimensional gravity. ${ }^{8}$ This and another interesting topic — the coupling of matter consistently with the

gauge principle ${ }^{9}$ - are beyond the scope of our review. In yet a further investigation one could study non-topological theories in which invariants (46) and/or (47) are added to the Lagrange density (43).

In conclusion, we note that dynamics determined by a group has been familiar in physics since the invention of Yang-Mills theory. However, the examples described here offer a new possibility: in the Lie algebra that determines a gauge theory one can allow an extension. This gives rise to richer dynamics within the same group theoretical structure, and in the gravity model studied above produces the cosmological constant.

\section{ACKNOWLEDGEMENTS}

The review was prepared with the assistance of D. Cangemi, particularly in finding the explicit solutions; this I gratefully acknowledge. 


\section{REFERENCES}

1. G. Dzhordzhadze, A. Pogrebkov and M. Polivanov, "General Solutions of the Cauchy Problem for the Liouville Equation $\varphi_{t t}(t, x)-\varphi_{x x}(t, x)=(1 / 2) m \exp \varphi(t, x)$," Dokl. Akad. Nauk. SSSR 243, 318 (1978) [Sov. Phys. Dokl. 23, 828 (1978)]; "Singular Solutions of the Equation $\square \varphi+\left(m^{2} / 2\right) \exp \varphi=0$ and Dynamics of Singularities," Teor. Mat. Fiz. 40, 221 (1979) [Theor. Math. Phys. 40, 706 (1979)].

2. E. D'Hoker and R. Jackiw, "Classical and Quantal Liouville Field Theory," Phys. Rev. D 26, 3517 (1982) and "Space-Translation Breaking and Compactification in the Liouville Theory," Phys. Rev. Lett. 50, 1719 (1983); E. D'Hoker, D. Freedman and R. Jackiw, "SO(2+1)-Invariant Quantization of the Liouville Theory," Phys. Rev. D 28, 2583 (1983).

3. C. Teitelboim, "Gravitation and Hamiltonian Structure in Two Space-Time Dimensions," Phys. Lett. 126B, 41 (1983) and "The Hamiltonian Structure of Two-Dimensional SpaceTime and its Relation with the Conformal Anomaly," in Quantum Theory of Gravity, S. Christensen, ed. (Adam Hilger, Bristol, 1984); R. Jackiw, "Liouville Field Theory: A Two-Dimensional Model for Gravity?," in Quantum Theory of Gravity, S. Christensen, ed. (Adam Hilger, Bristol, 1984) and "Lower-Dimensional Gravity," Nucl. Phys. B252, $343(1985)$.

4. H. Verlinde, "Black Holes and Strings in Two Dimensions," in The Sixth Marcel Grossman Meeting on General Relativity, H. Sato, ed. (World Scientific, Singapore, 1992); C. Callan, S. Giddings, A. Harvey and A. Strominger, "Evanescent Black Holes," Phys. Rev. D 45, 1005 (1992). Quantization of the model is discussed in these papers. 
5. T. Fukuyama and K. Kamimura, "Gauge Theory of Two-Dimensional Gravity," Phys. Lett. 160B, 259 (1985); K. Isler and C. Trugenberger, "Gauge Theory of TwoDimensional Quantum Gravity," Phys. Rev. Lett. 63, 834 (1989); A. Chamseddine and D. Wyler, "Gauge Theory of Topological Gravity in $1+1$ Dimensions," Phys. Lett. B 228, 75 (1989). Quantization of the model is discussed in these papers.

6. D. Cangemi and R. Jackiw, "Gauge Invariant Formulations of Lineal Gravity," Phys. Rev. Lett. (in press).

7. A. Achúcarro and P. Townsend, "A Chern-Simons Action for Three-Dimensional antide Sitter Supergravity Theories," Phys. Lett. B 180, 89 (1986); E. Witten, "2 + 1Dimensional Gravity as an Exactly Solvable System," Nucl. Phys. B311, 46 (1988/89).

8. D. Cangemi, in preparation.

9. G. Grignani and G. Nardelli, in preparation. 